\title{
Simulations Illustrate Flaw in Inflation Models
}

\author{
Peter L. D’Antonio, Ph.D. \\ Molloy College \\ Division of Business \\ USA
}

\begin{abstract}
This study questions the basic assumption of standard inflation models that there are only two forces driving price changes - underlying inflation and short-term noise. Specifically, the paper analyzes the distributions of individual price changes to shed light on an apparent contradiction: individual price changes are extremely dispersed, while measured inflation, the aggregate of individual price changes, remains relatively steady. Monte Carlo simulations demonstrate that the standard inflation model is inconsistent with this observation. The author reintroduces a new inflation model that explicitly accounts for the possibility of a third price-change driverlong-term sector-specific forces. Simulations show that the new three-forces model corresponds to the data quite well. If standard inflation measures, such as the CPI, are based on an incorrect two-forces model, then Fed policy based on those measures could be misguided. Inflation measures must explicitly account for the possibility that long-term sector-specific forces drive individual price changes.
\end{abstract}

Keywords: inflation, consumer price index, inflation measurement, monetary policy

\section{Introduction}

This paper follows D'Antonio (2017), which analyzed the distributions of individual price changes and found long-term sector-specific forces driving these price changes. That paper proposed a new inflation model that explicitly accounts for these forces. The current study extends that work to show that the standard model is inconsistent with the actual distribution of individual price changes, while the new model fits the data well. This study uses Monte Carlo simulations to show that the extreme dispersion of individual price changes is not a random occurrence, directly contradicting the assumption in standard inflation models. If it were, measured inflation itself, which combines these individual price changes, would exhibit much more volatility. Instead, the simulations demonstrate that the new model proposed in D'Antonio (2017), which allows for long-term sectorspecific forces driving price changes, is consistent with actual price change dispersion. This provides further evidence that the basic two-forces assumption of inflation is incorrect and that inflation models must allow for the possibility that other forces are at work.

Section 2 of this paper details past efforts to measure inflation under standard inflation model assumptions. Section 3 shows that individual prices are extremely widely dispersed, suggesting a high degree of volatility in price changes that is out of step with the relative stability of overall inflation measures. Section 4 presents the Monte Carlo simulations, which show that the distribution of actual inflation is inconsistent with the assumptions set out in standard inflation models. Section 5 lays out the new three-forces inflation model and uses the same simulations to demonstrate that actual inflation is consistent with this new model of inflation. Section 6 offers concluding remarks about inflation modeling and Fed policy.

\section{Inflation measurement}

The concept of inflation is different from the measurement of inflation. True inflation (the concept) is the rise in the general price level that simultaneously erodes the value of what a dollar can buy. This inflation concept has two important characteristics: (1) all prices rise together, since they are affected by the same common monetary force, and (2) these price increases are persistent and not short-term noise. In other words, the Fed injects more money into the economy than is needed to buy all goods and services, so each dollar becomes less valuable and the price level rises. 
The theoretical basis for all inflation measures is the following: There are just two forces that drive every individual price change $\left(\pi_{\mathrm{i}}\right)$ - an underlying inflation rate common to all prices $\left(\pi^{*}\right)$ and a short-term random noise term $\left(\mu_{\mathrm{i}}\right)$. Therefore, individual price changes can be modeled by the equation

(1) $\quad \pi_{\mathrm{i}}=\pi^{*}+\mu_{\mathrm{i}}$

When all these individual price changes are cumulated into a single measure such as the CPI or the PCE deflator, it can be written as

(2) $\quad \sum \pi_{\mathrm{i}}=\pi^{*}+\sum \mu_{\mathrm{i}}$

where $\sum \pi_{\mathrm{i}}$ is the weighted average of individual price changes, $\pi^{*}$ is the underlying inflation rate, and $\sum \mu_{\mathrm{i}}$ is the weighted average of the short-term noise. ${ }^{1}$ This last term is assumed to have two important special properties: zero mean and a normal distribution. So, on average the weighted average of the error terms equals zero, which in turn means that the inflation measure $\sum \pi_{\mathrm{i}}$ equals $\pi^{*} . \pi^{*}$ is what the inflation gauge is meant to measure, and thus forms the logic behind all inflation measures.

Unfortunately, it is impossible to simply measure changes in the general price level because it is unobservable. No statistic exists that measures only the price changes that are both persistent and common to all prices. Instead, the inflation rate must be estimated using data, which can fluctuate wildly in the short term. Measured inflation captures all price movements regardless of the reason for, or the duration of, the price changes. Economists have long wrestled with how to combine prices of different items with different values, some of which are rising fast, some slow, and some falling, to best estimate the inflation rate. Different inflation measures use different formulas, weighting schemes, and scopes. ${ }^{2}$ None of these measures adequately isolates the true underlying inflation rate from short-term noise.

Most inflation studies have focused on cutting through the short-term noise in inflation measurement by eliminating obvious sources of volatility in certain sectors. Most often, food and energy have been excluded from the inflation measure to get at what is known as a "core" reading. Sometimes, tobacco or owners' equivalent rent are excluded as well. Other efforts to reduce volatility include taking the median price or trimmed means (Bryan and Cicchetti, 1994; Cicchetti, 1996). Rich and Steindel (2007) reviewed all these "core" measures and found that although they succeeded in lowering volatility, no single measure was superior to the others. For purposes of monitoring inflation, the Fed uses both the PCE deflator and the deflator excluding food and energy.

Along with D'Antonio (2017), this study takes a completely different angle on inflation measurement by looking at the distributions of 50 individual prices that make up the CPI and the PCE deflator. This sector-by-sector approach, which has not been examined in the Economics literature, sheds light on two important questions with implications for understanding the inflation process, inflation measurement, and policy. These questions address (1) the amount of signal versus noise in individual price changes, and (2) whether the standard inflation model is consistent with the apparent volatility in individual prices. Based on this approach, these studies contend that the basic premise of the standard model that guides all inflation measures - that only two forces influence individual price changes - is incorrect.

\section{Signal versus noise ... or something else altogether}

D'Antonio (2018) looked at a cross section of individual price changes to determine the split between signal and noise. According to the standard inflation model, the only reason for price changes to diverge from the underlying path $\pi^{*}$ is due to short-term noise $\mu_{\mathrm{i}}$. So that paper calculated the divergence of each price change $\pi_{\mathrm{i}}$ from the mean price change (proxy for $\pi^{*}$ ) to see if it rose along with the underlying path of inflation $\pi^{*}$.

The price change would suggest more signal than noise if it was close to the underlying inflation path, and more noise than signal if it was far from the underlying path. This exercise was conducted to assess the accuracy of current inflation measures - more signal would mean more precise measures, while more noise would mean less precise measures.

\footnotetext{
${ }^{1}$ As an example, the Fed's Underlying Inflation Gauge in Amistad (2014) uses this specification of the standard inflation model.

${ }^{2}$ The CPI is calculated using a Laspeyres index, while the PCE deflator uses a Fisher Ideal index. The CPI weights are based on the Consumer Expenditure Survey, which measures quantities of consumer spending, while the PCE deflator bases the weighting on what households actually consume (regardless of who pays for it). The differences in these estimates are detailed in McCully, Moyer and Stewart (2007).
} 


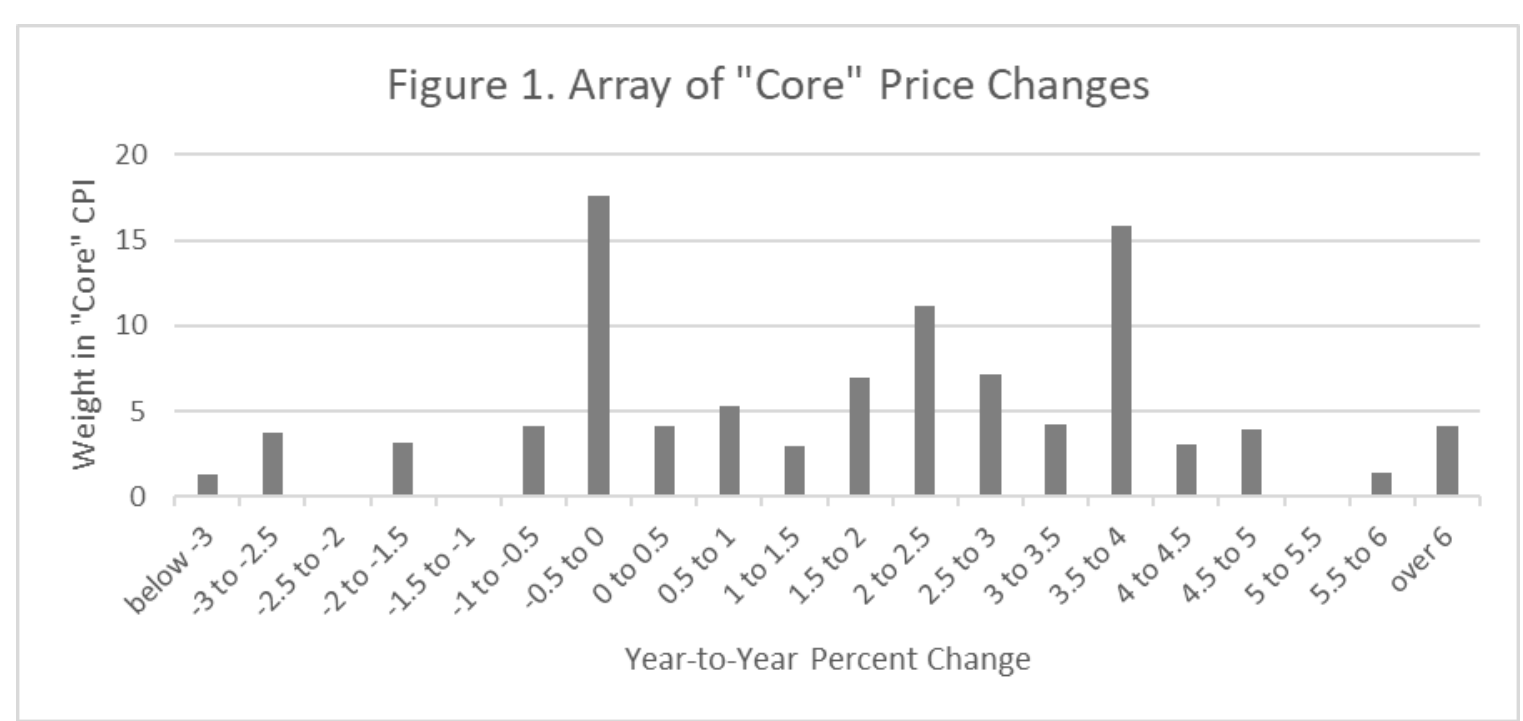

If the signal was strong ( $\pi^{*}$ is the main driver), all the prices should be bunched together; if the noise was strong ( $\mu_{\mathrm{i}}$ is the main driver), the prices should be widely dispersed. As it turned out, the individual price changes are very widely dispersed. Figure 1 shows a histogram of all price changes, taking out three obvious sources of noise: food, energy, and owners' equivalent rent. ${ }^{3}$ The horizontal axis shows an array of various size price changes, and the vertical bars represent the weight of the CPI that exhibited those price changes. Price changes stretched from 3 percent to +6 percent. Importantly, only 28 percent of price changes were within a percentage point of the Fed's 2 percent inflation target. The wide dispersion was typical of other years over the past two decades as well.

While these data seem to suggest that short-term volatility $\mu_{\mathrm{i}}$ is very large, indicating more noise than signal, an altogether different phenomenon could be at work. One implication of volatile price changes is that the aggregated measured inflation rate based on those volatile price changes should be volatile as well. However, the opposite occurs - inflation is traditionally one of the more stable economic indicators. This inconsistency challenges our understanding of why prices change and implies that the two-forces standard inflation model is flawed.

\section{Monte Carlo simulations highlight inconsistency}

Given the apparent volatility in individual price changes, this section aims to determine if the wide dispersion could be consistent with the relative stability of overall inflation measures. One way to make this determination is to simulate inflation measurements using the statistical properties of individual prices under the assumption that any divergence from the mean must be short-term random noise. These simulations can be compared with actual inflation measurements.

Specifically, the implied volatility of the inflation measure is found by simulating $\mu_{\mathrm{i}}$ using Equation 1 (taking $\pi_{\mathrm{i}}-$ $\left.\pi^{*}\right)$ and cumulating the error terms, $\sum \mu_{\mathrm{i}}$. The study assumes that, for each of the 50 items in the core inflation measure of Section 3 , the $\mu_{\mathrm{i}}$ is normally distributed, with zero mean, and variance is given by the actual variance over the past 20 years, denoted $\sigma_{\mathrm{i}}$.

Using a random number generator and these assumptions, as well as the assumption in the standard inflation model that there are only two forces driving individual prices, the study makes 100 simulations of 20 versions of the $50 \mu_{\mathrm{i}}$ error terms. ${ }^{4}$ These values are then multiplied by the CPI weights of the 50 items studied and cumulated using a Laspeyres formula to get a weighted average of the $\mu_{\mathrm{i}}$ for each of the 20 years, $\sum \mu_{\mathrm{i}}$. In other words, these simulations yielded an array of 100 sets of 20 weighted averages of short-term noise, which can be used to compare with the actual inflation measures over the past 20 years. The study isolated the residual noise that comes through in the simulated CPI measure based on the actual variances of the individual price changes. Figure 2 shows the distribution of the standard deviations of these 100 sets of weighted averages of short-term noise.

\footnotetext{
${ }^{3}$ Source data for the analysis and figures in this study are from the Bureau of Labor Statistics and Haver Analytics. The data in Figure 1 show the dispersion of price changes over the year from May 2017 to May 2018.

${ }^{4}$ The random numbers are found using the built-in random number generator in Excel, which is expressed as Rand() and then converted to fit into a normal distribution with zero mean and $\sigma_{\mathrm{i}}$ using $\operatorname{Norminv}\left(\operatorname{Rand}(), 0, \sigma_{\mathrm{i}}\right)$. 
In general, these standard deviations averaged about 0.66 , with values rarely reaching as low as 0.45 or as high as $0.86 .{ }^{5}$ In other words, these simulations suggest that actual inflation should have a standard deviation of about 0.66 each year, if the standard inflation model holds, using the actual volatility of individual price changes.

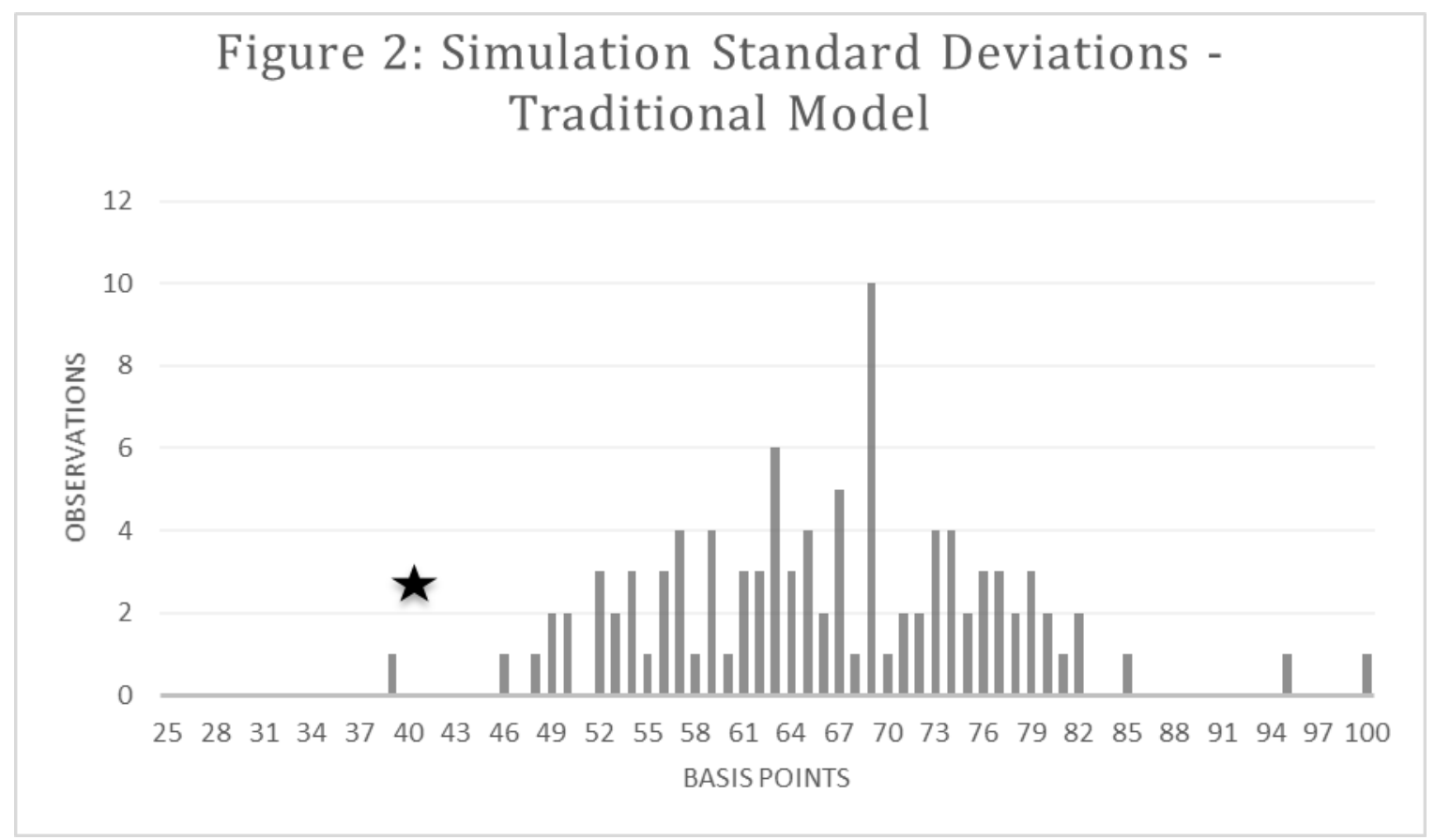

This value seems quite high, as measured inflation has tended to be much more stable than implied by these simulations. The standard deviation of the 20 years of actual price change data is just 0.40 , using the same expenditure items, weights, and formula as the simulations. This actual value, indicated by the star in Figure 2, is clearly an outlier. It is unlikely to be generated by the same forces and processes as are assumed in the simulations. In 100 trials, there was only one instance in which the simulation gave results in the same range as the actual standard deviation. This result suggests that the basic assumptions in the inflation model are wrong.

\section{New inflation model gives consistent results}

This paper finds that the divergences between individual price changes are very large, implying that inflation measures should be much more volatile than is actually the case. This puzzling result may be clarified by altering the assumption that there are only two forces driving price changes. What if multiple sector-specific forces drove long-term price changes as well?

D'Antonio (2017) proposed a new inflation model in which long-term sector-specific forces drive individual sector prices. This new model can be written

(3) $\quad \pi_{\mathrm{i}}=\pi^{*}+\rho_{\mathrm{i}}+\mu_{\mathrm{i}}$

where $\rho_{\mathrm{i}}$ represents forces that influence sector i price changes on a long-term basis. This is the same equation as (1) except for the added term $\rho_{\mathrm{i}}$. If this new model is correct, the divergence from the underlying inflation rate, $\pi_{\mathrm{i}}$ $-\pi^{*}$, would be caused by both long-term sector-specific price changes, $\rho_{\mathrm{i}}$, and short-term random noise, $\mu_{\mathrm{i}}$.

The existence of long-term forces other than underlying inflation can help explain the dichotomy between the wide dispersion from the mean of individual price changes and the relative stability of current price measures. In other words, individual price changes which are far from $\pi^{*}$ might not represent random noise at all. If the divergences are the product of long-term forces, the price changes would be expected to remain far from $\pi^{*}$ year after year, but not contribute to the volatility of the overall inflation measure. This can be tested using the same simulations as in Section 4 - with one adjustment.

\footnotetext{
${ }^{5}$ Only one observation of the 100 simulations posted a standard deviation below 0.45 and only two posted standard deviations above 0.86 .
} 
Instead of basing the standard errors on the difference between $\pi_{\mathrm{i}}$ and $\pi^{*}$, which according to Equation 3 would include both short-term noise and long-term sectoral differences $\left(\pi_{i}-\pi^{*}=\rho_{i}+\mu_{i}\right)$, the standard errors can be based on the difference between $\pi_{\mathrm{i}}$ and the expected value of $\pi_{\mathrm{i}}\left(=\pi^{*}+\rho_{\mathrm{i}}\right)$, which would represent only short-term noise $\left(\pi_{\mathrm{i}}-\pi^{*}-\rho_{\mathrm{i}}=\mu_{\mathrm{i}}\right)^{6}$

\section{Figure 3. Simulation Standard Deviation - Refined Model}

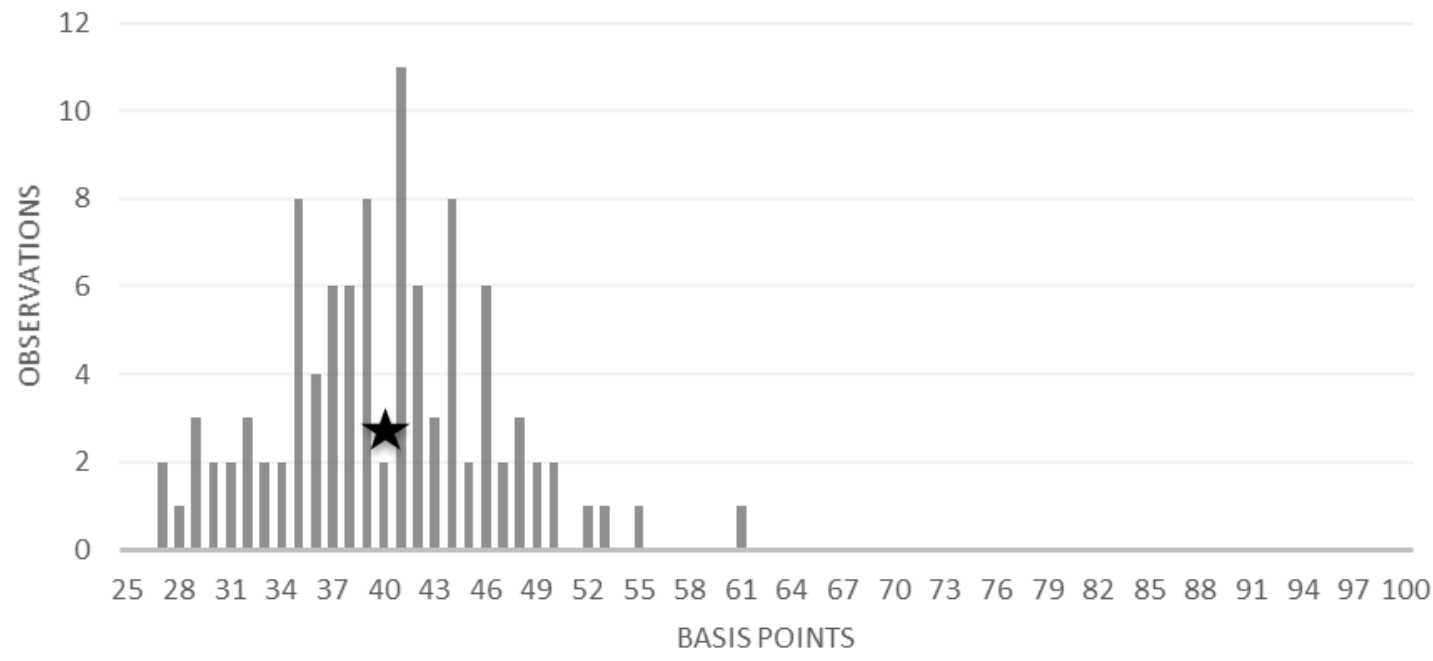

Using the same simulation trials and weights, but different standard errors that capture variances in individual prices relative to their own averages, a new set of 100 simulations was generated. This new array of simulated weighted averages $\sum \mu_{\mathrm{i}}$ shows significantly lower standard errors (see Figure 3 ). The mean standard error is close to 0.40 , which is the standard error of the actual average price changes in the calculated core CPI (the star in Figure 3). Actual price change data fit much more closely with this new model, which allows for the existence of multiple forces affecting individual sector prices.

\section{Conclusion}

These results suggest that standard inflation modeling, along with inflation measures based on this modeling, is incorrect. Simulations in this paper showed that the implied standard errors (differences between individual price changes and average price changes) are too large to be consistent with actual measured inflation. However, when the simulations include the possibility of long-term forces driving price changes, the simulated standard errors become consistent with actual data.

The evidence indicates that effective inflation modeling needs to explicitly allow for other forces besides underlying inflation and short-term noise to influence price changes, along the lines of the new inflation model proposed by D'Antonio (2017). Failing to explicitly include these other forces risks the Fed systematically pursuing policies that are ineffectual or even counterproductive.

\section{References}

Amstad, M., Potter, S., \& Rich, R. (2014). The FRBNY Staff Underlying Inflation Gauge: UIG. Federal Reserve Bank of New York Staff Report No. 672.

Bryan, M. F., \& Cecchetti, S. G. (1994). Measuring Core Inflation. In N. G. Mankiw (Ed.), Monetary Policy (195215). Chicago: University of Chicago Press.

Bureau of Labor Statistics, CPI-All Urban Consumers data.

Cecchetti, S. G. (1996). Measuring Short-Run Inflation for Central Bankers. National Bureau of Economic Research Working Paper No. 5786.

\footnotetext{
${ }^{6}$ The temporary noise terms $\left(\mu_{\mathrm{i}}\right)$ of each individual price $\mathrm{i}$ in the new model of inflation were approximated by the difference between the actual price change each year $\left(\pi_{\mathrm{i}}\right)$ and the average price change of $\mathrm{i}$ (over 20 years). 
D’Antonio, P. L. (2017). Are Inflation Measures Really Measuring Inflation? Journal of Business and Economic Policy, 4 (4).

D'Antonio, P. L. (2018). Wide Dispersion of Price Changes Limit the Accuracy of Inflation Measures. Northeast Decision Science Institute Conference Proceedings, April 2018.

Haver Analytics consumer price index data.

Khan, M., Morel L., \& Sabourin, P. (2013). The Common Component of CPI: An Alternative Measure of Underlying Inflation for Canada. Bank of Canada Working Paper No. 2013-35.

McCully, C. P., Moyer, B. C., \& Stewart, K. J. (2007). A Reconciliation Between the Consumer Price Index and the Personal Consumption Expenditure Price Index. Bureau of Economic Analysis.

Rich, R., and Steindel, C. (2007). A Comparison of Measures of Core Inflation. Federal Reserve Bank of New York, Economic Policy Review 13(3). 19-38. 